\title{
An Elementary Proof of the Existence and Uniqueness Theorem for the Navier-Stokes Equations
}

\author{
J. C. Mattingly*and Ya. G. Sinai ${ }^{\dagger}$ \\ November 19, 1998, Revised March 10th, 1999
}

\section{Introduction}

The purpose of this paper is to show that some results concerning solutions of the Navier-Stokes systems can be proven by purely elementary methods. In two-dimensions with periodic boundary conditions, the Navier-Stokes system has the form

$$
\begin{aligned}
\frac{\partial u_{1}}{\partial t}+u_{1} \frac{\partial u_{1}}{\partial x_{1}}+u_{2} \frac{\partial u_{1}}{\partial x_{2}} & =\nu \Delta u_{1}-\frac{\partial p}{\partial x_{1}}+f_{1}\left(x_{1}, x_{2}, t\right) \\
\frac{\partial u_{2}}{\partial t}+u_{1} \frac{\partial u_{2}}{\partial x_{1}}+u_{2} \frac{\partial u_{2}}{\partial x_{2}} & =\nu \Delta u_{2}-\frac{\partial p}{\partial x_{2}}+f_{2}\left(x_{1}, x_{2}, t\right), \\
\frac{\partial u_{1}}{\partial x_{1}}+\frac{\partial u_{2}}{\partial x_{2}} & =0 .
\end{aligned}
$$

Here $\nu$ is the viscosity, $p$ is the pressure, and $f_{1}, f_{2}$ are the components of an external forcing which may be time-dependent. As our setting is periodic, the functions $u_{1}, u_{2}, \nabla p, f_{1}$, and $f_{2}$ are all periodic in $x$. For simplicity, we take the period to be one.

The first existence and uniqueness theorems for weak solutions of (1) were proven by Leray (Ler34) in whole plane $\mathbb{R}^{2}$. Later these results were extended by E. Hopf (see [Hop51). In 1962, Ladyzenskaya proved existence and uniqueness results for strong solutions for general two-dimensional domains Lad69. V. Yudovich, C. Foias, R. Teman, P. Constantin, and others developed strong methods which provided deep insights into the dynamics described by (11) (see [Yud89, Tem79, Tem95, CF88]).

The purpose of this paper is to present elementary proofs of three theorems. These theorems imply the existence and uniqueness of smooth solutions of (1) and shed some additional light on the dissipative character of the dynamics. We will also discuss what our techniques can give in the three-dimensional setting.

In two-dimensions, it is useful to consider the vorticity $\omega\left(x_{1}, x_{2}, t\right)=\frac{\partial u_{1}\left(x_{1}, x_{2}, t\right)}{\partial x_{2}}-\frac{\partial u_{2}\left(x_{1}, x_{2}, t\right)}{\partial x_{1}}$. The equation governing $\omega$ has the form ( see CM93, DG95)

$$
\frac{\partial \omega}{\partial t}+u_{1} \frac{\partial \omega}{\partial x_{1}}+u_{2} \frac{\partial \omega}{\partial x_{2}}=\nu \Delta \omega+g\left(x_{1}, x_{2}, t\right)
$$

where $g\left(x_{1}, x_{2}, t\right)=\frac{\partial f_{1}\left(x_{1}, x_{2}, t\right)}{\partial x_{2}}-\frac{\partial f_{2}\left(x_{1}, x_{2}, t\right)}{\partial x_{1}}$. We will need $g\left(x_{1}, x_{2}\right)$ to posses a modicum of spatial smoothness; this will be made precise shortly.

In our two-dimensional setting, the systems (11) and (2) are equivalent. Expanding $\omega$ in Fourier series where $\omega\left(x_{1}, x_{2}, t\right)=\sum_{k \in \mathbb{Z}^{2}} \omega_{k}(t) e^{2 \pi i(x, k)}$ with $x=\left(x_{1}, x_{2}\right)$, we can write a coupled ODE-system for the modes $\omega_{k}(t)$ (see DG95).

$$
\frac{d \omega_{k}}{d t}+2 \pi i \sum_{l_{1}+l_{2}=k} \omega_{l_{1}} \omega_{l_{2}} \frac{\left(k, l_{2}^{\perp}\right)}{\left(l_{2}, l_{2}\right)}=-4 \pi^{2} \nu|k|^{2} \omega_{k}+g_{k}(t)
$$

\footnotetext{
*Department of Mathematics, Stanford University, Stanford CA 94305.

$\dagger$ Department of Mathematics, Princeton University, Princeton NJ 08544.
} 
where $k \in \mathbb{Z}^{2},|k|=\sqrt{k_{1}^{2}+k_{2}^{2}}, l^{\perp}=\left(l^{(1)}, l^{(2)}\right)^{\perp}=\left(-l^{(2)}, l^{(1)}\right)$, and $g_{k}(t)$ are the spatial Fourier modes of the function $g(x, t)$. Since $\omega$ is real, we know $\omega_{-k}=\bar{\omega}_{k}$. Furthermore, we always assume that $\omega_{0}=0$. The system (3) is the Galerkin system corresponding to (2). A finite dimensional approximation of this Galerkin system can be associated to any finite subset $\mathcal{Z}$ of $\mathbb{Z}^{2}$ by setting $\omega_{k}(t)=0$ for all $k$ outside of $z$. In the following, we will implicitly assume that $Z$ is centrally-symmetric, that is if $k \in \mathcal{Z}$ then $-k \in \mathcal{Z}$.

In fact, we will study a slightly more general version of (2) where the Laplacian is replaced by the operator $|\nabla|^{\alpha}$ with $\alpha>1$. This leads to a version of (3) which we index by the choice of $\alpha$ and by the finite index set $z, z \subset \mathbb{Z}^{2}$, indicating which modes are included in the Galerkin approximation. In short, we consider the finite dimensional ODE system

$$
\frac{d \omega_{k}}{d t}+2 \pi i \sum_{\substack{l_{1}+l_{2}=k \\ l_{1}, l_{2} \in \mathcal{Z}}} \omega_{l_{1}} \omega_{l_{2}} \frac{\left(k, l_{2}^{\perp}\right)}{\left(l_{2}, l_{2}\right)}=-4 \pi^{2} \nu|k|^{\alpha} \omega_{k}+g_{k} .
$$

We now state the assumptions on the coefficients $g_{k}(t)$ to be used at various times during our discussion.

Assumption 1. The forcing $f(x, t)=\left(f_{1}(x, t), f_{2}(x, t)\right)$ is such that $g^{*}=\sup _{t \in[0, \infty)}|g(\cdot, t)|_{L^{2}}<\infty$.

Assumption 2. For some $r$, there exists a constant $\mathcal{G}(r)>0$ such that

$$
\sup _{t \in[0, \infty)}\left|g_{k}(t)\right| \leq \frac{\mathcal{G}(r)}{|k|^{r-\alpha+\epsilon}}
$$

for some $\epsilon>0$ and all $k \in \mathbb{Z}^{2} \backslash 0$. The constant $\alpha$ is the same as in $\left(\mathbb{G}_{2}^{\alpha}\right)$.

Assumption 3. For some $r$ and $\gamma>0$, there exists a constant $\mathcal{G}(r, \gamma)>0$ such that

$$
\sup _{t \in[0, \infty)}\left|g_{k}(t)\right| \leq \frac{\mathcal{G}(r, \gamma)}{|k|^{r-\alpha+\epsilon}} e^{-\gamma|k|^{1+\delta}}
$$

for some $\delta>0, \epsilon>0$, and all $k \in \mathbb{Z}^{2} \backslash 0$. Again, the constant $\alpha$ is the same as in ( $\left(\begin{array}{l}\alpha \\ \alpha\end{array}\right)$.

Observe that assumption 3 implies assumption 2. Critical to our discussion is that for (3 $3 \%$ ) we have the so-called enstrophy estimate. Namely, if $\mathcal{E}(0)=\int \omega^{2}\left(x_{1}, x_{2}, 0\right) d x_{1} d x_{2}=\sum_{k}\left|\omega_{k}(0)\right|^{2}<\infty$ then one can find $\mathcal{E}^{*}$ depending only on $\mathcal{E}(0), \nu, \sup _{t \in[0, \infty)}|g(\cdot, t)|_{L^{2}}$, and $\alpha$ such that $\mathcal{E}(t)=\int \omega^{2}\left(x_{1}, x_{2}, t\right) d x_{1} d x_{2} \leq \mathcal{E}^{*}$ for all solutions to $\left(33_{z}^{\alpha}\right)$. It is important to note that $\mathcal{E}^{*}$ is independent of the set $z$ which defines the Galerkin approximation. This enstrophy estimate holds if the forcing satisfies assumption 1 (see e.g. CF88, DG95, Tem79).

Now we are ready to formulate our theorems.

Theorem 1. Assume the forcing satisfies assumption 1 and 国 for some $r>1$ and $\mathcal{G}(r)>0$. If for some $\mathcal{D}_{1}<\infty$

$$
\left|\omega_{k}(0)\right| \leq \frac{\mathcal{D}_{1}}{|k|^{r}}
$$

then one can find a $\mathcal{D}_{1}^{\prime}<\infty$, depending only on $\mathcal{D}_{1}, \nu, g^{*}$, and $\mathcal{G}$, such that any solution to $\left(\mathcal{G}_{z}^{\alpha}\right)$ with these initial conditions satisfies

$$
\left|\omega_{k}(t)\right| \leq \frac{\mathcal{D}_{1}^{\prime}}{|k|^{r}}
$$

for all $t>0$. In particular, $\mathcal{D}_{1}^{\prime}$ is independent of the set $z$ defining the Galerkin approximation.

An existence and uniqueness theorem for (3) follows from theorem 11 by now standard considerations (see CF88, DG95, Tem79). We briefly recall the general line of the argument. By the Sobolev embedding theorem, the Galerkin approximations are trapped in a compact subset of $L^{2}$ of the 2-torus. This guarantees the existence of a limit point which can be shown to satisfy (3). Using the the regularity inherited from the Galerkin approximations, one then shows that there is a unique solution to (3). Gallavotti [Gal96] contains a similar proof of a similar statement. 
Theorem 2. Assume that assumption $\mathrm{g}$ holds for some $r>1, \gamma>0$, and $\mathcal{G}(r, \gamma)>0$. If the initial conditions satisfy

$$
\left|\omega_{k}(0)\right| \leq \frac{\mathcal{D}_{2}}{|k|^{r}} e^{-\gamma_{2}|k|}
$$

for some $\mathcal{D}_{2}<\infty$ and $\gamma_{2}>0$, then one can find a $\mathcal{D}_{2}^{\prime}<\infty$ and a $\gamma_{2}^{\prime}>0$, depending only on $\mathcal{D}_{2}, \gamma, r, \nu$, $g^{*}, \mathcal{G}$, such that any solution to (画) starting from these initial conditions satisfies

$$
\left|\omega_{k}(t)\right| \leq \frac{\mathcal{D}_{2}^{\prime}}{|k|^{r}} e^{-\gamma_{2}^{\prime}|k|}
$$

for all $t>0$. In particular, the constants $\mathcal{D}_{2}^{\prime}$ and $\gamma^{\prime}$ are independent of the set $\mathcal{z}$ defining the Galerkin approximation.

Theorem 2 shows that equation (2) preserves the class of real analytic functions on the 2-torus.

Theorem 3. Assume that assumption $\mathrm{G}$ holds for some $r>1, \gamma>0$, and $\mathcal{G}(r, \gamma)>0$. If the initial conditions satisfy

$$
\left|\omega_{k}(0)\right| \leq \frac{\mathcal{D}_{3}}{|k|^{r}}
$$

then for any $t_{0}>0$, one can find $a \mathcal{D}_{3}^{\prime}>0$ and $a \gamma_{3}^{\prime}>0$ such that any solution to (摖) with these initial conditions satisfies

$$
\left|\omega_{k}\left(t_{0}\right)\right| \leq \frac{\mathcal{D}_{3}^{\prime}}{|k|^{r}} e^{-\gamma_{3}^{\prime}|k|}
$$

As before, the constant $\mathcal{D}_{3}^{\prime}$ is independent of the set $z$ defining the Galerkin approximation.

Theorem 3 shows that if the initial conditions $\omega(x, 0)$ for (2) are smooth enough then, the solution $\omega\left(x, t_{0}\right)$ is real analytic for arbitrarily small time $t_{0}$. . Then according to theorem 2, it remains with in this class for all $t>t_{0}$. Statements close to these were proven in the works by C. Foias and R. Temam [FT89], C. Doering and E. Titi [DT95] and H. Kreiss [Kre88]. Theorem 1 is proven in $\S 2$ and theorems 2 and 3 are proven in 83 .

The proofs of all of the theorems in this paper share a common structure. We consider the system of coupled ODEs for the Fourier coefficients. Then we construct a subset $\Omega$ of the phase space (the set of possible configurations of the Fourier modes) so that all points in $\Omega$ possess the desired decay properties. In addition, $\Omega$ is constructed so that it contains the initial data in its interior. Then we endeavor to show that the dynamics never cause the sequence of Fourier modes to leave the subset $\Omega$. How this is done can be understood geometrically. It amounts to showing that the vector field on the boundary of $\Omega$ points into the interior of $\Omega$. If this is true, then the solution can never escape $\Omega$.

\section{Proof of Theorem 1}

Fixing an arbitrary Galerkin approximation corresponding to the modes in some finite subset $z$ of $\mathbb{Z}^{2}$, we write the real version of (3). As we already mentioned, we assume $\omega_{0}=0$ and, because the velocity is real, we also have $\omega_{-k}=\bar{\omega}_{k}$. Setting $\omega_{k}=\omega_{k}^{(1)}+i \omega_{k}^{(2)}$, we separate the equations for $\omega_{k}^{(1)}$ and $\omega_{k}^{(2)}$ obtaining

$$
\begin{aligned}
& \frac{d \omega_{k}^{(1)}}{d t}=2 \pi \sum_{\substack{l_{1}+l_{2}=k \\
l_{1}, l_{2} \in \mathcal{Z}}}\left[\omega_{l_{1}}^{(1)} \omega_{l_{2}}^{(2)}+\omega_{l_{1}}^{(2)} \omega_{l_{2}}^{(1)}\right] \frac{\left(k, l_{2}^{\perp}\right)}{\left(l_{2}, l_{2}\right)}-4 \pi \nu|k|^{\alpha} \omega_{k}^{(1)}+g_{k}^{(1)} \\
& \frac{d \omega_{k}^{(2)}}{d t}=-2 \pi \sum_{\substack{l_{1}+l_{2}=k \\
l_{1}, l_{2} \in \mathcal{Z}}}\left[\omega_{l_{1}}^{(1)} \omega_{l_{2}}^{(1)}+\omega_{l_{1}}^{(2)} \omega_{l_{2}}^{(2)}\right] \frac{\left(k, l_{2}^{\perp}\right)}{\left(l_{2}, l_{2}\right)}-4 \pi \nu|k|^{\alpha} \omega_{k}^{(2)}+g_{k}^{(2)}
\end{aligned}
$$


where $g_{k}=g_{k}^{(1)}+i g_{k}^{(2)}$.

It follows from the enstrophy estimate that $\sum_{k}\left[\left(w_{k}^{(1)}(t)\right)^{2}+\left(w_{k}^{(2)}(t)\right)^{2}\right] \leq \mathcal{E}^{*}$ and thus $\left|w_{k}^{(1)}(t)\right| \leq \sqrt{\mathcal{E}^{*}}$ and $\left|w_{k}^{(2)}(t)\right| \leq \sqrt{\mathcal{E}^{*}}$ for all $k \in \mathbb{Z}^{2}$ and $t>0$. Hence, for any $K_{0}>0$, we can find a $\mathcal{D}_{1}^{\prime}=\mathcal{D}_{1}^{\prime}\left(K_{0}\right)$ such that for any $t \geq 0\left|w_{k}^{(1)}(t)\right|,\left|w_{k}^{(2)}(t)\right|<\frac{\mathcal{D}_{1}^{\prime}}{|k|^{r}}$ for all $k \in \mathbb{Z}^{2}$ with $|k| \leq K_{0}$. We also require $\mathcal{D}_{1}^{\prime}$ to be greater than $\mathcal{G}$ so later estimates will arrange themselves nicely. Recall that $\mathcal{G}(r)$ was the constant from assumption 2. Since $\mathcal{G}$ is given and only $K_{0}$ is ours to vary, we will suppress the dependence of $\mathcal{D}_{1}^{\prime}$ on $\mathcal{G}$.

Now consider the subset

$$
\Omega_{1}\left(K_{0}\right)=\left\{\left(\omega_{k}^{(1)}, \omega_{k}^{(2)}\right)_{k \in \mathbb{Z}^{2}}:\left|\omega_{k}^{(j)}\right| \leq \frac{\mathcal{D}_{1}^{\prime}\left(K_{0}\right)}{|k|^{r}} \text { for all } j \in\{1,2\}, k \in \mathbb{Z}^{2} \backslash 0\right\}
$$

of $\left(\mathbb{R}^{2}\right)^{\mathbb{Z}^{2}}$. Its boundary is the subset

$$
\partial \Omega_{1}\left(K_{0}\right)=\left\{\left(\omega_{k}^{(1)}, \omega_{k}^{(2)}\right)_{k \in \mathbb{Z}^{2}}: \begin{array}{c}
\left|\omega_{k}^{(j)}\right| \leq \frac{\mathcal{D}_{1}^{\prime}}{|k|^{r}} \text { for all } j \in\{1,2\}, k \in \mathbb{Z}^{2} \backslash 0 \\
\text { and equality holds for some } \bar{k} \text { and } \bar{\jmath} .
\end{array}\right\} .
$$

We shall also need the subset of this boundary

$$
\overline{\partial \Omega_{1}}\left(K_{0}\right)=\left\{\left(\omega_{k}^{(1)}, \omega_{k}^{(2)}\right)_{k \in \mathbb{Z}^{2}}: \quad \begin{array}{c}
\left|\omega_{k}^{(j)}\right| \leq \frac{\mathcal{D}_{1}^{\prime}}{|k|^{r}} \text { for all } j \in\{1,2\}, k \in \mathbb{Z}^{2} \backslash 0 \\
\text { and equality for some } \bar{k} \text { and } \bar{\jmath} \text { with }|\bar{k}|>K_{0} .
\end{array}\right\} .
$$

Showing that the trajectories of our system remain inside of $\Omega_{1}$ is equivalent to the statement of the theorem. Recall that using the enstrophy estimate, we picked a $\mathcal{D}_{1}^{\prime}\left(K_{0}\right)$ such that if $|k| \leq K_{0}$ then $\left|w_{k}^{(1)}(t)\right|$ and $\left|w_{k}^{(2)}(t)\right|$ were bounded by $\frac{\mathcal{D}_{1}^{\prime}}{|k|^{r}}$ for all $t \in[0, \infty)$. Thus, the only remaining way for a trajectory to leave $\Omega_{1}\left(K_{0}\right)$, is through the section of the boundary $\overline{\partial \Omega_{1}}\left(K^{0}\right)$ introduced above. Our basic idea is to show that if $K_{0}$ is greater than a specific $K_{\text {crit }}$, then the vector field on $\overline{\partial \Omega_{1}}\left(K^{0}\right)$ points inward. In other words, the dynamics of (伍) can never move the system configuration through $\partial \Omega_{1}\left(K_{0}\right)$. In still different words, $\Omega_{1}$ is a trapping region. Since the initial data begins in $\Omega_{1}$, proving this picture would prove the theorem.

To show that the vector field points inward, fix a point on $\overline{\partial \Omega_{1}}\left(K_{0}\right)$. For definiteness, consider the case when $\omega_{\bar{k}}^{(1)}=\frac{\mathcal{D}_{1}^{\prime}}{|k|^{r}}$ for some $\bar{k}$ with $|\bar{k}|>K_{0},\left|\omega_{k^{\prime}}^{(1)}\right| \leq \frac{\mathcal{D}_{1}^{\prime}}{\left|k^{\prime}\right|^{r}}$ for all $k^{\prime} \in \mathbb{Z} \backslash 0$ with $k^{\prime} \neq \bar{k}$, and $\left|\omega_{k}^{(2)}\right| \leq \frac{\mathcal{D}_{1}^{\prime}}{|k|^{r}}$ for all $k \in \mathbb{Z}^{2} \backslash 0$. The other cases, namely where $\omega_{\bar{k}}^{(1)}=-\frac{\mathcal{D}_{1}^{\prime}}{|k|^{2}}$ or $\omega_{\bar{k}}^{(2)}= \pm \frac{\mathcal{D}_{1}^{\prime}}{|k|^{2}}$, are handled in the same manner. We have to show that,

$$
2 \pi\left|\sum_{l_{1}+l_{2}=\bar{k}}\left[\omega_{l_{1}}^{(1)} \omega_{l_{2}}^{(1)}+\omega_{l_{1}}^{(2)} \omega_{l_{2}}^{(2)}\right] \frac{\left(\bar{k}, l_{2}^{\perp}\right)}{\left(l_{2}, l_{2}\right)}\right|+\left|g_{\bar{k}}^{(2)}\right|<4 \pi \nu|\bar{k}|^{\alpha}\left|\omega_{\bar{k}}^{(2)}\right| .
$$

We shall see that the restriction that $|\bar{k}| \geq K_{0}>K_{\text {crit }}$ does not depend on $\mathcal{D}_{1}$ only on $\mathcal{E}$. (5):

Consider the following three sums which together bound the first abolute value on the left-hand side of

$$
\begin{aligned}
& \Sigma_{1}=\sum_{\substack{l_{1}+l_{2}=\bar{k} \\
\left|l_{2}\right| \leq\left|\overline{\frac{k}{2}}\right|}}\left|\left[\omega_{l_{1}}^{(1)} \omega_{l_{2}}^{(1)}+\omega_{l_{1}}^{(2)} \omega_{l_{2}}^{(2)}\right]\right|\left|\frac{\left(\bar{k}, l_{2}^{\perp}\right)}{\left(l_{2}, l_{2}\right)}\right| \\
& \Sigma_{2}=\sum_{\substack{l_{1}+l_{2}=\bar{k} \\
\left|\frac{\bar{k}}{2}\right|<\left|l_{2}\right| \leq 2|\bar{k}|}}\left|\left[\omega_{l_{1}}^{(1)} \omega_{l_{2}}^{(1)}+\omega_{l_{1}}^{(2)} \omega_{l_{2}}^{(2)}\right]\right|\left|\frac{\left(\bar{k}, l_{2}^{\perp}\right)}{\left(l_{2}, l_{2}\right)}\right| \\
& \Sigma_{3}=\sum_{\substack{l_{1}+l_{2}=\bar{k} \\
\left|l_{2}\right|>2|\bar{k}|}}\left|\left[\omega_{l_{1}}^{(1)} \omega_{l_{2}}^{(1)}+\omega_{l_{1}}^{(2)} \omega_{l_{2}}^{(2)}\right]\right|\left|\frac{\left(\bar{k}, l_{2}^{\perp}\right)}{\left(l_{2}, l_{2}\right)}\right|
\end{aligned}
$$


We treat each sum separately. For $\Sigma_{1}$, using the Cauchy-Schwartz inequality and the inequalities $\left|\frac{\left(\bar{k}, l_{2}\right)}{\left(l_{2}, l_{2}\right)}\right| \leq \frac{|\bar{k}|}{\left|l_{2}\right|}$, $\left|l_{1}\right| \geq \frac{|\bar{k}|}{2}$, and $\left|\omega_{l_{1}}^{(1)}\right| \leq 2^{r} \mathcal{D}_{1}^{\prime} \frac{1}{|k|^{r}},\left|\omega_{l_{1}}^{(2)}\right| \leq 2^{r} \mathcal{D}_{1}^{\prime} \frac{1}{|k|^{r}}$ produces

$$
\begin{aligned}
\left|\Sigma_{1}\right| & \leq 2^{r} \frac{\mathcal{D}_{1}^{\prime}}{|\bar{k}|^{r}}|\bar{k}| \sum_{\left|l_{2}\right| \geq\left|\frac{\bar{k}}{2}\right|}\left[\left|\omega_{l_{2}}^{(1)}\right|+\left|\omega_{l_{2}}^{(2)}\right|\right] \frac{1}{\left|l_{2}\right|} \\
& \leq 2^{r} \frac{\mathcal{D}_{1}^{\prime}|\bar{k}|}{|\bar{k}|^{r}}\left(\sqrt{\sum\left|\omega_{l_{2}}^{(1)}\right|^{2}}+\sqrt{\sum\left|\omega_{l_{2}}^{(2)}\right|^{2}}\right) \sqrt{\sum_{\left|l_{2}\right| \leq\left|\frac{\bar{k}}{2}\right|} \frac{1}{\left|l_{2}\right|^{2}}} \\
& \leq 2^{r+1} \text { (const) }\left(\sqrt{\mathcal{E}^{*}}\right)|\bar{k}|(\sqrt{\ln |\bar{k}|})\left(\frac{\mathcal{D}_{1}^{\prime}}{|\bar{k}|^{r}}\right) .
\end{aligned}
$$

The (const) in the final line is from the inequality

$$
\sum_{\left|l_{2}\right| \leq\left|\frac{\bar{k}}{2}\right|} \frac{1}{\left|l_{2}\right|^{2}} \leq(\text { const })^{2} \ln |\bar{k}| .
$$

To estimate $\Sigma_{2}$, we use the inequalities $\left|\frac{\left(k, l_{2}^{\perp}\right)}{\left(l_{2}, l_{2}\right)}\right| \leq 2,\left|\omega_{l_{2}}^{(1)}\right| \leq 2^{r} \frac{\mathcal{D}_{1}^{\prime}}{|k|^{r}}$, and $\left|\omega_{l_{2}}^{(2)}\right| \leq 2^{r} \frac{\mathcal{D}_{1}^{\prime}}{|k|^{r}}$ obtaining

$$
\begin{aligned}
\left|\Sigma_{2}\right| & \leq 2^{r+1} \frac{\mathcal{D}_{1}^{\prime}}{|\bar{k}|^{r}} \sum_{\left|l_{1}\right| \leq 3|\bar{k}|}\left[\left|\omega_{l_{1}}^{(1)}\right|+\left|\omega_{l_{1}}^{(2)}\right|\right] \\
& \leq 2^{r+1} \frac{\mathcal{D}_{1}^{\prime}}{|\bar{k}|^{r}}\left[\sqrt{\sum_{\left|l_{1}\right| \leq 3|\bar{k}|}\left|\omega_{l_{1}}^{(1)}\right|^{2}}+\sqrt{\sum_{\left|l_{1}\right| \leq 3|\bar{k}|}\left|\omega_{l_{1}}^{(2)}\right|^{2}}\right](6|\bar{k}|+1) \\
& \leq 2^{r+2} \mathcal{E}(6|\bar{k}|+1) \frac{\mathcal{D}_{1}^{\prime}}{|\bar{k}|^{r}} .
\end{aligned}
$$

The factor $(6|\bar{k}|+1)$ arises as an estimate of the square root of the number of lattice points $l_{1} \in \mathbb{Z}^{2}$ for which $\left|l_{1}\right| \leq 3|\bar{k}|$.

In estimating $\Sigma_{3}$, we use $\left|\frac{\left(\bar{k}, l_{2}^{\perp}\right)}{\left(l_{2}, l_{2}\right)}\right| \leq\left|\frac{\bar{k}}{l_{2}}\right|$ producing

$$
\begin{aligned}
\left|\Sigma_{3}\right| & \leq|\bar{k}| \sum_{\substack{l_{1}+l_{2}=\bar{k} \\
\left|l_{2}\right| \geq|\bar{k}|}}\left[\left|\omega_{l_{1}}^{(1)}\right|\left|\omega_{l_{2}}^{(2)}\right|+\left|\omega_{l_{1}}^{(2)}\right|\left|\omega_{l_{2}}^{(1)}\right|\right] \frac{1}{\left|l_{2}\right|} \\
& \leq|\bar{k}|\left[\left(\sum_{\left|l_{1}\right| \geq \bar{k}}\left(\omega_{l_{1}}^{(1)}\right)^{2}\right)^{\frac{1}{2}}\left(\sum_{\left|l_{2}\right| \geq 2 \bar{k}} \frac{\left(\omega_{l_{2}}^{(2)}\right)^{2}}{\left|l_{2}\right|^{2}}\right)^{\frac{1}{2}}+\left(\sum_{\left|l_{1}\right| \geq \bar{k}}\left(\omega_{l_{1}}^{(2)}\right)^{2}\right)^{\frac{1}{2}}\left(\sum_{\left|l_{2}\right| \geq 2 \bar{k}} \frac{\left|\omega_{l_{2}}^{(1)}\right|^{2}}{\left|l_{2}\right|}\right)^{\frac{1}{2}}\right] \\
& \leq 2 \sqrt{\mathcal{E}^{*}}|\bar{k}| \mathcal{D}_{1}^{\prime}\left(\sum_{\left|l_{2}\right| \geq 2|\bar{k}|} \frac{1}{\left|l_{2}\right|^{2(r+1)}}\right)^{\frac{1}{2}} \leq 2 \sqrt{\mathcal{E}^{*}}(\text { const })|\bar{k}| \frac{\mathcal{D}_{1}^{\prime}}{|\bar{k}|^{r}}
\end{aligned}
$$

where (const) is defined by the inequality

$$
\sum_{\left|l_{2}\right| \geq 2|\bar{k}|} \frac{1}{\left|l_{2}\right|^{2(r+1)}} \leq(\text { const })^{2} \frac{1}{|\bar{k}|^{2 r}} .
$$

Adding (6), (7), and (8) together, we obtain the needed bound on the right hand side of (5): 


$$
\begin{aligned}
2 \pi \sum_{l_{1}+l+2=\bar{k}}\left|\omega_{l_{1}}^{(1)}\right|\left|\omega_{l_{2}}^{(2)}\right|+\left|\omega_{l_{1}}^{(2)}\right|\left|\omega_{l_{2}}^{(1)}\right| \leq & {\left[2^{r+1}(\text { const }) \sqrt{\mathcal{E}^{*}}|\bar{k}| \sqrt{\ln |\bar{k}|}+2^{r+2} \mathcal{E}^{*}(6|\bar{k}|+1)\right.} \\
& \left.+2 \sqrt{\mathcal{E}^{*}}(\text { const })|k|\right] \frac{\mathcal{D}_{1}^{\prime}}{|\bar{k}|^{r}} \\
\leq & 2^{r+2} \mathcal{E}^{*}(\overline{\text { const }})|\bar{k}| \sqrt{\ln |\bar{k}|} \frac{\mathcal{D}_{1}^{\prime}}{|\bar{k}|^{r}}
\end{aligned}
$$

where $(\overline{\text { const }})$ is a new constant.

By assumption 2 and our requirement that the $\mathcal{D}_{1}^{\prime}$ be greater than $\mathcal{G}$ (the constant from assumption 2), we know that $\left|g_{k}\right| \leq \frac{\mathcal{D}_{1}^{\prime}}{|k|^{r-\alpha+\epsilon}}$. Thus, inequality (5) will be satisfied if

$$
\left[2^{r+2} \mathcal{E}^{*}(\overline{\text { const }}) \frac{|\bar{k}| \sqrt{\ln |\bar{k}|}}{|\bar{k}|^{\alpha}}+\frac{1}{|\bar{k}|^{\epsilon}}\right] \frac{\mathcal{D}_{1}^{\prime}}{|\bar{k}|^{r-\alpha}} \leq 4 \pi \nu \frac{\mathcal{D}_{1}^{\prime}}{|\bar{k}|^{r-\alpha}} .
$$

From this we see that for all $\alpha>1$, there exists $K_{\text {crit }}$ so that if $|\bar{k}| \geq K_{\text {crit }}$ then (10) holds. Also notice that $K_{\text {crit }}$ is independent of our choice of $\mathcal{D}_{1}^{\prime}$ except for the condition that $\mathcal{D}_{1}^{\prime}>\mathcal{G}$. Thus we can find $K_{\text {crit }}$ first and then fix $K_{0}$ which determines $\mathcal{D}_{1}^{\prime}$.

\section{Proofs of Theorems 2 and 3}

We begin by stating the central estimate on which both theorems rely. It requires estimates similar in spirit to the previous theorem and will be proven at the end of the section. We present a $d$-dimensional version of the lemma because it will be useful in the discussions of the 3-dimensional setting in the next section.

Lemma 1. Let $\left\{a_{k}\right\}$ and $\left\{b_{k}\right\}$ be two sequences with $k \in \mathbb{Z}^{d}$. If for some $r>d-1$ and some $\mathcal{C}>0$

$$
\left|a_{k}\right| \leq \frac{\mathcal{C}}{|k|^{r}} \quad\left|b_{k}\right| \leq \frac{\mathcal{C}}{|k|^{r}}
$$

then for all $k \in \mathbb{Z}^{d}$

$$
\sum_{\substack{l_{1}+l_{2}=k \\ l_{1}, l_{2} \in \mathbb{Z}^{d}}}\left|a_{l_{1}}\right|\left|b_{l_{2}}\right| \frac{|k|}{\left|l_{2}\right|} \leq(\text { const })\left(2^{r}|k|+2^{r+1}(6|k|+1)^{\frac{d}{2}}+\frac{1}{2}|k|^{d-1-r}\right) \frac{\mathcal{C}^{2}}{|k|^{r}}
$$

where the constant depends only on $r$ and not on $k$.

We now turn to the proof of theorem 2 .

Proof of theorem 目. If $\left|\omega_{k}^{(1)}(0)\right| \leq \frac{\mathcal{D}_{2}}{|k|^{r}} e^{-\gamma_{2}|k|},\left|\omega_{k}^{(2)}(0)\right| \leq \frac{\mathcal{D}_{2}}{|k|^{r}} e^{-\gamma_{2}|k|}$ then surely $\left|\omega_{k}^{(1)}\right|,\left|\omega_{k}^{(2)}\right| \leq \frac{\mathcal{D}_{2}}{|k|^{r}}$. Therefore by theorem 1 , one can find a constant $\overline{\mathcal{D}}_{2}$ such that $\left|\omega_{k}^{(1)}(t)\right|,\left|\omega_{k}^{(2)}(t)\right| \leq \frac{\overline{\mathcal{D}}_{2}}{|k|^{r}}$ for all $k \in \mathbb{Z}^{2} \backslash\{0\}$. Let us set $\mathcal{D}_{2}^{\prime}=\max \left(2 \overline{\mathcal{D}}_{2}, \mathcal{G}\right)$ where $\mathcal{G}$ is the constant from assumption 3 . The numerical factor 2 is somewhat arbitrary. We could chose any factor greater than 1 ; we take 2 for simplicity.

Choose $K_{0} \geq 0$ and consider the set

$$
\Omega_{2}\left(K_{0}\right)=\left\{\left(\omega_{k}^{(1)}, \omega_{k}^{(2)}\right)_{k \in \mathbb{Z}^{2} \backslash\{0\}}:\left|\omega_{k}^{(1)}\right| \leq \frac{\mathcal{D}_{2}^{\prime}}{|k|^{r}} e^{-\gamma_{2}^{\prime}|k|},\left|\omega_{k}^{(2)}\right| \leq \frac{\mathcal{D}_{2}^{\prime}}{|k|^{r}} e^{-\gamma_{2}^{\prime}|k|}\right\}
$$

The value of $\gamma_{2}^{\prime}=\gamma_{2}^{\prime}\left(K_{0}\right)$ is chosen in such a way that the inequalities $\left|\omega_{k}^{(i)}(t)\right| \leq \frac{\overline{\mathcal{D}}_{2}}{|k|^{r}}$ given by theorem 1 imply that $\left|\omega_{k}^{(i)}(t)\right| \leq \frac{\mathcal{D}_{2}^{\prime}}{|k|^{r}} e^{-\gamma_{2}^{\prime}|k|}$ for all $k,|k| \leq K_{0}$, and that for $|k| \geq K_{0}, e^{-\gamma_{2}^{\prime}|k|} \geq e^{-\gamma|k|^{1+\delta}}$. Here $\gamma$ and $\delta$ are the constants from assumption 3 . 
As in the proof of theorem it, we shall show that for sufficiently large $K_{0}$ the vector field corresponding to (5) is directed inside $\Omega_{2}\left(K_{0}\right)$ along the part of the boundary $\partial \Omega_{2}\left(K_{0}\right)$ where $\left|\omega_{k}^{(i)}\right| \leq \frac{\mathcal{D}_{2}^{\prime}}{|k|^{r}} e^{-\gamma_{2}^{\prime}|k|}$ for all $k \in \mathbb{Z}^{2} \backslash\{0\}$ with $|k| \geq K_{0}$ and for at least one of these, say $\bar{k}$, we have equality. It will be shown that our restriction from below on $K_{0}$, needed to ensure the vector field points inward, will not depend on $\gamma_{2}^{\prime}$. This will yield the stated result.

As in theorem 1, consider for definiteness the case where $\omega_{\bar{k}}^{(1)}=\frac{\mathcal{D}_{2}^{\prime}}{|k|^{2}} e^{-\gamma_{2}^{\prime}|\bar{k}|}$. The other cases are handled in the same manner. As before, we have to show that the vector field points inward. This would be assured if

$$
2 \pi\left|\sum_{l_{1}+l_{2}=\bar{k}}\left[\omega_{l_{1}}^{(1)} \omega_{l_{2}}^{(2)}+\omega_{l_{2}}^{(2)} \omega_{l_{2}}^{(1)}\right] \frac{\left(\bar{k}, l_{2}\right)}{\left(l_{2}, l_{2}\right)}\right|+\left|g_{\bar{k}}\right|<4 \pi^{2}|\bar{k}|^{\alpha} \frac{\mathcal{D}_{2}^{\prime}}{|\bar{k}|^{r}} e^{-\gamma_{2}^{\prime}|\bar{k}|}
$$

This time we do not use the enstrophy estimate as previously. Instead, we use the estimates $\left|\omega_{l_{1}}^{(1)}\right| \leq$ $\frac{\mathcal{D}_{2}^{\prime}}{\left|l_{1}\right|^{r}} e^{-\gamma_{2}^{\prime}\left|l_{1}\right|}$ and $\left|\omega_{l_{1}}^{(2)}\right| \leq \frac{\mathcal{D}_{2}^{\prime}}{\left|l_{2}\right|^{r}} e^{-\gamma_{2}^{\prime}\left[\left|l_{2}\right|\right.}$.

Let us put $v_{k}^{(j)}=e^{\gamma_{2}^{\prime}|k|} \omega_{k}^{(j)}, j=1,2, k \in \mathbb{Z}^{2} \backslash 0$. In terms of $v$, (11) becomes

$$
2 \pi\left|\sum_{l_{1}+l_{2}=\bar{k}}\left[v_{l_{1}}^{(1)} v_{l_{2}}^{(2)}+v_{l_{2}}^{(2)} v_{l_{2}}^{(1)}\right] \frac{e^{-\gamma_{2}^{\prime}\left|l_{1}\right|-\gamma_{2}^{\prime}\left|l_{2}\right|}}{e^{-\gamma_{2}^{\prime}|\bar{k}|}} \frac{\left(\bar{k}, l_{2}^{\perp}\right)}{\left(l_{2}, l_{2}\right)}\right|+\left|g_{\bar{k}}\right| e^{\gamma_{2}^{\prime}|\bar{k}|}<4 \pi^{2}|\bar{k}|^{\alpha} \frac{\mathcal{D}_{2}^{\prime}}{|\bar{k}|^{r}} .
$$

First notice that $\frac{e^{-\gamma_{2}^{\prime}\left|l_{1}\right|} e^{-\gamma_{2}^{\prime}\left|l_{2}\right|}}{e^{-\gamma_{2}^{\prime}|k|}} \leq 1$ so it may be neglected. Second notice that for $v_{k}^{(j)}$, we have the estimate $\left|v_{k}^{(j)}\right| \leq \frac{\mathcal{D}_{2}^{\prime}}{|k|^{r}}$ for $k \in \mathbb{Z}^{2} \backslash 0$. Lastly, we know that $\left|\frac{\left(\bar{k}, l_{2}\right)}{\left(l_{2}, l_{2}\right)}\right| \leq \frac{|k|}{\left|l_{2}\right|}$. These estimates allow us to apply lemma 1, producing

$$
\begin{aligned}
2 \pi \mid \sum\left[v_{l_{1}}^{(1)} v_{l_{2}}^{(2)}+v_{l_{2}}^{(2)} v_{l_{2}}^{(1)}\right] & \frac{e^{-\gamma_{2}^{\prime}\left|l_{1}\right|-\gamma_{2}^{\prime}\left|l_{2}\right|}}{e^{-\gamma_{2}^{\prime}|\bar{k}|}} \frac{\left(\bar{k}, l_{2}^{\perp}\right)}{\left(l_{2}, l_{2}\right)} \mid \\
& \leq 2 \pi \text { const }\left(2^{r+1}|\bar{k}|+2^{r+2}(6|\bar{k}|+1)+|\bar{k}|^{1-r}\right) \mathcal{D}_{2}^{\prime} \frac{\mathcal{D}_{2}^{\prime}}{|\bar{k}|^{r}} .
\end{aligned}
$$

From this estimate, we see that if

$$
2 \pi \text { (const) }\left(2^{r+1}|\bar{k}|+2^{r+2}(6|\bar{k}|+1)+2|\bar{k}|^{1-r}\right) \mathcal{D}_{2}^{\prime}+\frac{\mathcal{G}}{\mathcal{D}_{2}^{\prime}} \frac{e^{-\gamma|\bar{k}|^{1+\delta}}}{e^{-\gamma_{2}^{\prime}|\bar{k}|}}|\bar{k}|^{\alpha-\epsilon}<4 \pi^{2} \nu|\bar{k}|^{\alpha}
$$

then we have established (12), which was our goal. Notice that we chose $\mathcal{D}_{2}^{\prime} \geq \mathcal{G}$ and $\gamma_{2}^{\prime}$ such that $\frac{e^{-\gamma|\bar{k}| 1+\delta}}{e^{-\gamma_{2}^{\prime}|k|} \leq 1}$ for all $k$ with $|k| \geq K_{0}$. Since $\alpha>1$ by picking $K_{0}$ large enough, we can force (14) to hold. This is the criteria which sets the level of $K_{\text {crit }}$. The proof of theorem 2 is concluded.

We now present the proof of theorem 3. Its structure is very similar to the previous proof and also employs lemma 11 but uses a slightly different change of variable.

Proof of theorem S. Let $\mathcal{D}_{1}^{\prime}$ be the constant given by theorem 1 that is such that $\left|\omega_{k}(t)\right| \leq \frac{\mathcal{D}_{1}^{\prime}}{|k|^{2}}$ for all $k \in \mathbb{Z}^{2} \backslash 0$ and all $t$. Let us put $v_{k}^{(j)}=\omega_{k}^{(j)} e^{\gamma_{3} t|k|}, j=1,2$ where the constant $\gamma_{3}$ will be determined later. The evolution of the $v_{k}^{(1)}(t)$ are described by the following ODEs

$$
\begin{aligned}
\frac{d v_{k}^{(1)}(t)}{d t}= & \gamma_{3}|k| v_{k}^{(1)}(t)-4 \pi^{2} \nu|k|^{\alpha} v_{k}^{(1)}(t)+g_{k}^{(1)} e^{\gamma_{3} t|k|} \\
& -2 \pi \sum_{l_{1}+l_{2}=k}\left[v_{l_{1}}^{(1)}(t) v_{l_{2}}^{(2)}(t)+v_{l_{1}}^{(2)}(t) v_{l_{2}}^{(1)}(t)\right] \frac{e^{-\gamma_{3} t\left|l_{1}\right|} e^{-\gamma_{3} t\left|l_{2}\right|}}{e^{-\gamma_{3} t|k|}} \frac{\left(k, l_{2}^{\perp}\right)}{\left(l_{2}, l_{2}\right)} .
\end{aligned}
$$


The analogous equations describe the evolution of the $v_{k}^{(2)}(t)$.

The methods of the previous section can be applied to this coupled system. We fix a time $t_{0}>0$ and an arbitrary positive constant $\gamma_{0}$. For $t=0$, we have the inequalities

$$
\left|v_{k}^{(1)}(0)\right| \leq \frac{\mathcal{D}_{3}}{|k|^{r}} \quad\left|v_{k}^{(2)}(0)\right| \leq \frac{\mathcal{D}_{3}}{|k|^{r}}
$$

for all $k$. In light of the definition of $v_{k}(t)$, theorem 3 would be proven if we show that

$$
\left|v_{k}^{(1)}\left(t_{0}\right)\right| \leq \frac{\mathcal{D}_{3}^{\prime}}{|k|^{r}} \quad\left|v_{k}^{(2)}\left(t_{0}\right)\right| \leq \frac{\mathcal{D}_{3}^{\prime}}{|k|^{r}}
$$

for some appropriate $\mathcal{D}_{3}^{\prime}$.

As in the proof of theorem 3 , we put $\mathcal{D}_{3}^{\prime}=\max \left(2 \mathcal{D}_{1}^{\prime}, \mathcal{G}\right)$ where $\mathcal{G}$ is again the constant from assumption 3. For any fixed $K_{0}$, we can find a $\gamma_{3}$ so that the following three conditions hold. First, the inequalities $\left|\omega_{k}^{(j)}(t)\right| \leq \frac{\mathcal{D}_{1}^{\prime}}{|k|^{r}}$, imply $\left|v_{k}^{(j)}(t)\right| \leq \frac{\mathcal{D}_{3}^{\prime}}{|k|^{r}}$ for $j=1,2, t \in\left[0, t_{0}\right]$, and $|k| \leq K_{0}$. Second, so $e^{\gamma_{3} t|k|} \leq e^{\gamma|k|^{1+\delta}}$ for $k \in \mathbb{Z}^{2}$ with $|k| \geq K_{0}$ and $t \in\left[0, t_{0}\right]$. In this condition the constants $\gamma$ and $\delta$ are again from assumption 3 . Third, we can always assume that $\gamma_{3} \leq \gamma_{0}$. (This last assumption is to simplify the exposition and is not really needed as $\gamma_{3}$ decreases as we increase $K_{0}$.)

Now consider the set

$$
\Omega_{3}\left(K_{0}\right)=\left\{\left(v_{k}^{(1)}, v_{k}^{(2)}\right)_{k \in \mathbb{Z}^{2} \backslash 0} \text { with }\left|v_{k}^{(j)}\right| \leq \frac{\mathcal{D}_{3}^{\prime}}{|k|^{r}} \text { for } j=1,2 \text { and }|k|>K_{0}\right\} .
$$

Again we will show that if $K_{0}$ is greater than some $K_{\text {crit }}$, the vector field along the boundary of $\Omega_{3}\left(K_{0}\right)$ points inward. The calculation parallels that in theorem 2. For definiteness, we assume that $v_{\bar{k}}^{(1)}(t)=\frac{\mathcal{D}_{3}^{\prime}}{|k|^{r}}$ for some $\bar{k}$ with $|\bar{k}|>K_{0}$ and that the inequality bounds which define $\Omega_{3}$ hold for all other $k$. The other cases proceed analogously.

We wish to show that the vector field points inward. Since $\gamma_{3} \leq \gamma_{0}$, from (15), we see that it is sufficient to show that for $t \in\left[0, t_{0}\right]$

$$
\begin{aligned}
\left(4 \pi^{2} \nu|\bar{k}|^{\alpha}-\gamma_{0}|\bar{k}|\right) v_{\bar{k}}^{(1)}>2 & \left|\sum_{l_{1}+l_{2}=\bar{k}}\left[v_{l_{1}}^{(1)}(t) v_{l_{2}}^{(2)}(t)+v_{l_{1}}^{(2)}(t) v_{l_{2}}^{(1)}(t)\right] \frac{\left(k, l_{2}\right)}{\left(l_{2}, l_{2}\right)}\right| \\
& +\left|g_{\bar{k}}^{(1)}\right| e^{\gamma_{3} t|\bar{k}|} .
\end{aligned}
$$

Here, as before, we have neglected the factor $\frac{e^{-\gamma_{3} t\left|l_{1}\right|} e^{-\gamma_{3} t\left|l_{2}\right|}}{e^{-\gamma_{3} t|k|}}$ as it is always less than 1 . After applying the inequalities $\mathcal{G} \leq \mathcal{D}_{3}^{\prime}, e^{\gamma_{3} t|k|} \leq e^{\gamma|k|^{1+\delta}}$ and lemma 1, we see that (17) holds if

$$
4 \pi^{2} \nu>\gamma_{0} \frac{|\bar{k}|}{|\bar{k}|^{\alpha}}+\text { (const) } \mathcal{D}_{3}^{\prime}\left[2^{r+1} \frac{|\bar{k}|}{|\bar{k}|^{\alpha}}+2^{r+2} \frac{7|\bar{k}|}{|\bar{k}|^{\alpha}}+\frac{|\bar{k}|^{1-r}}{|\bar{k}|^{\alpha}}\right]+\frac{\mathcal{G}}{\mathcal{D}_{3}^{\prime}} \frac{1}{|\bar{k}|^{\alpha}} .
$$

Because $\alpha>1$ and $r>2$, by making $\bar{k}$ large enough we can force (18) to hold. This shows that the solution to any Galerkin approximation stays in $\Omega_{3}$ until the time $t_{0}$ and thus (16) holds and the proof is complete.

Proof of Lemma 1: As in the proof of theorem 1, we estimate separately three sums.

$$
\begin{aligned}
& \Sigma_{1}=\sum_{\substack{\left|l_{2}\right| \leq\left|\frac{k}{2}\right| \\
l_{1}+l_{2}=k}}\left|a_{l_{1}}\right|\left|b_{l_{2}}\right| \frac{|k|}{\left|l_{2}\right|} \\
& \Sigma_{2}=\sum_{\substack{\left|\frac{k}{\mid}\right|<\left|l_{2}\right| \leq 2|k| \\
l_{1}+l_{2}=k}}\left|a_{l_{1}}\right|\left|b_{l_{2}}\right| \frac{|k|}{\left|l_{2}\right|} \\
& \Sigma_{3}=\sum_{\substack{\left|l_{2}\right|>2|k| \\
l_{1}+l_{2}=k}}\left|a_{l_{1}}\right|\left|b_{l_{2}}\right| \frac{|k|}{\left|l_{2}\right|}
\end{aligned}
$$


Since in $\Sigma_{1}$, the norm of $\left|l_{1}\right| \geq\left|\frac{\bar{k}}{2}\right|$, we can write

$$
\Sigma_{1} \leq \sum_{\left|l_{2}\right| \leq\left|\frac{k}{2}\right|}\left|a_{l_{1}}\right|\left|b_{l_{2}}\right| \frac{|k|}{\left|l_{2}\right|} \leq \frac{2^{r}(\mathcal{C})^{2}}{|k|^{r}}|k| \sum_{\left|l_{2}\right| \leq\left|\frac{k}{2}\right|} \frac{1}{\left|l_{2}\right|^{r+1}} \leq 2^{r} \text { (const) }|k| \frac{\mathfrak{C}^{2}}{|k|^{r}}
$$

where the constant is defined by the inequality

$$
\sum_{l_{2} \in \mathbb{Z}^{d} \backslash 0} \frac{1}{\left|l_{2}\right|^{r+1}} \leq \text { const } .
$$

For this sum to be finite, we need $r+1>d$. For $\Sigma_{2}$ we have $\frac{|k|}{\left|l_{2}\right|} \leq 2$ and hence

$$
\begin{aligned}
\Sigma_{2} & \leq 2 \sum_{\left|\frac{k}{2}\right|<\left|l_{2}\right| \leq 2|k|} \frac{\mathcal{C}^{2}}{\left|l_{1}\right|^{r}\left|l_{2}\right|^{r}} \leq \frac{2^{r+2}(\mathcal{C})^{2}}{|\bar{k}|^{r}} \sum_{\left|l_{1}\right| \leq 3|k|} \frac{1}{\left|l_{1}\right|^{r}} \\
& \leq \frac{2^{r+2}(\mathcal{C})^{2}}{|\bar{k}|^{r}}\left(\sum_{\left|l_{1}\right| \leq 3|k|} \frac{1}{\left|l_{1}\right|^{2 r}}\right)^{\frac{1}{2}}\left(\sum_{\left|l_{1}\right| \leq 3|k|} 1\right)^{\frac{1}{2}} \leq 2^{r+1} \text { (const) } \frac{\mathcal{C}^{2}(6|k|+1)}{|k|^{r}} .
\end{aligned}
$$

Here the constant is the absolute constant defined by

$$
\sum_{l_{1} \in \mathbb{Z}^{d} \backslash 0} \frac{1}{\left|l_{1}\right|^{2 r}} \leq \text { const }
$$

For this sum to be finite, we need $2 r>d$. For $\Sigma_{3}$ we have $\frac{|k|}{\left|l_{2}\right|} \leq \frac{1}{2}$. Hence, we can write

$$
\begin{aligned}
\Sigma_{3} & \leq \frac{1}{2} \sum_{\left|l_{2}\right| \geq 2|\bar{k}|} \frac{\mathrm{e}^{2}}{\left|l_{1}\right|^{r}\left|l_{2}\right|^{r+1}} \leq \frac{\mathrm{e}^{2}}{2}\left(\sum_{\substack{\left|l_{1}\right|>|k| \\
l_{1} \in \mathbb{Z}^{d} \backslash 0}} \frac{1}{\left|l_{1}\right|^{2 r}}\right)^{\frac{1}{2}}\left(\sum_{\substack{\left|l_{2}\right| \geq 2|k| \\
l_{2} \in \mathbb{Z}^{d} \backslash 0}} \frac{1}{\left|l_{2}\right|^{2 r+2}}\right)^{\frac{1}{2}} \\
& \leq \text { (const) } \frac{|k|^{d-1-r}}{2} \frac{\mathrm{e}^{2}}{|k|^{r}} .
\end{aligned}
$$

Collecting together (19),(20),(21), we obtain the lemma.

\section{The three-dimensional setting}

This paper is mainly concerned with presenting an elementary proof of existence and uniqueness results in the two-dimensional setting. However, these techniques can also be used to gain some insight into the three-dimensional setting. On the three torus, the Navier-Stokes equations take the form

$$
\begin{aligned}
\frac{\partial u_{i}}{\partial t}+\sum_{j=1,2,3} u_{j} \frac{\partial u_{i}}{\partial x_{j}} & =\nu \Delta u_{j}-\frac{\partial p}{\partial x_{i}}+f_{i} \quad i=1,2,3 \\
\sum_{i=1,2,3} \frac{\partial u_{i}}{\partial x_{i}} & =0
\end{aligned}
$$

where $\nu>0$ is again the viscosity, $p$ is the pressure, and the $f_{i}$ are the components of the external, timedependent forcing. As before, we introduce the vorticity $\omega(x, t)=\left(\omega_{1}(x, t), \omega_{2}(x, t), \omega_{3}(x, t)\right)=\left(\frac{\partial u_{2}}{\partial x_{3}}-\right.$ $\left.\frac{\partial u_{3}}{\partial x_{2}}, \frac{\partial u_{3}}{\partial x_{1}}-\frac{\partial u_{1}}{\partial x_{3}}, \frac{\partial u_{1}}{\partial x_{2}}-\frac{\partial u_{2}}{\partial x_{1}}\right)$. The vorticity obeys the equation

$$
\frac{\partial \omega_{i}}{\partial t}+\sum_{j} u_{j} \frac{\partial \omega_{i}}{\partial x_{j}}=\sum_{j} \omega_{j} \frac{\partial u_{i}}{\partial x_{j}}+\nu \Delta \omega_{i}+g_{i} \quad i=1,2,3
$$


where the $g_{i}$ are the components of curl $f$. Moving to Fourier space where

$$
u(x, t)=\sum_{k \in \mathbb{Z}^{3}} u_{k}(t) e^{2 \pi i(k, x)} \text { and } \omega(x, t)=\sum_{k \in \mathbb{Z}^{3}} \omega_{k}(t) e^{2 \pi i(k, x)},
$$

we obtain

$$
\frac{d \omega_{k}(t)}{d t}=-2 \pi i \sum_{l_{1}+l_{2}=k}\left[\left(u_{l_{1}}(t), l_{2}\right) \omega_{l_{2}}(t)-\left(\omega_{l_{1}}(t), l_{2}\right) u_{l_{2}}(t)\right]-4 \pi^{2} \nu|k|^{2} \omega_{k}(t)+g_{k}(t) .
$$

Here the $g_{k}(t)$ are the Fourier components of the forcing $g(x, t)$. In addition, we can replace the Laplacian with the more general differential operator $|\nabla|^{\alpha}$ with $\alpha>1$.

The incompressibility condition implies that

$$
u_{k}(t) \perp k
$$

for all $k \in \mathbb{Z}^{3}$. Similarly, it follows that $\omega_{k}(t) \perp k, \omega_{k}(t) \perp u_{k}(t)$, and $\left|\omega_{k}(t)\right|=|k|\left|u_{k}(t)\right|$. Hence, $\left(k, u_{k}, \omega_{k}\right)$ is a right-handed orthogonal (but not orthonormal) frame.

Since $\left(u_{l_{1}}(t), l_{1}\right)=\left(\omega_{l_{1}}(t), l_{1}\right)=0$, we can rewrite (24) as

$$
\frac{d \omega_{k}(t)}{d t}=-2 \pi i \sum_{l_{1}+l_{2}=k}\left[\left(u_{l_{1}}(t), k\right) \omega_{l_{2}}(t)-\left(\omega_{l_{1}}(t), k\right) u_{l_{2}}(t)\right]-4 \pi^{2} \nu|k|^{\alpha} \omega_{k}(t)+g_{k}(t) \text {. }
$$

As before, we begin by restricting our attention to a finite subset $z \subset \mathbb{Z}^{3}$. The finite-dimensional Galerkin system corresponding to $z$ is

$$
\frac{d \omega_{k}(t)}{d t}=-2 \pi i \sum_{\substack{l_{1}+l_{2}=k \\ l_{1}, l_{2} \in Z}}\left[\left(u_{l_{1}}(t), k\right) \omega_{l_{2}}(t)-\left(\omega_{l_{1}}(t), k\right) u_{l_{2}}(t)\right]-4 \pi^{2} \nu|k|^{\alpha} \omega_{k}(t)+g_{k}(t) \cdot\left(26 z_{z}^{\alpha}\right)
$$

Furthermore, to simplify the arguments, we assume that the forcing $g(x, t)$ is a trigonometric polynomial which implies that all but a finite number of the $g_{k}$ are identically zero. We will always analyze wave numbers above the band which is directly forced; hence, we may neglect the $g_{k}$. This is only for convenience. The forcing can be included in the same way as it was in the two-dimensional setting.

Our development is based upon the basic energy estimate (see CF88, DG95, Tem79). It states that given any initial data such that $\sum_{k \in \mathbb{Z}^{3}}\left|u_{k}(0)\right|^{2}=E_{0}<\infty$ then there exists a constant $E^{*}$ depending only on $E_{0}, \nu, \sup _{t}|g(\cdot, t)|_{L^{2}}$ such that for any finite-dimensional Galerkin approximation, defined by $Z \subset \mathbb{Z}^{3}$, we have $\sum_{k \in z}\left|u_{k}(t)\right|^{2}<E^{*}$ for all $t>0$.

When $\alpha=2$, the system $(26 \%)$ corresponds to the Navier-Stokes equations. Unfortunately, we are unable to prove the theorems in this setting analogous to theorems 1, 2, and 3 when $\alpha=2$. However, if we increase $\alpha$, we can.

Theorem 4. Consider the system (20 2 ) with an $\alpha>2.5$ and satisfying assumption $\mathrm{O}$. If the initial data $\left\{\omega_{k}(0)\right\}$ are such that

$$
\left|\omega_{k}(0)\right| \leq \frac{\mathcal{D}_{4}}{|k|^{r}}
$$

for all $k \in \mathbb{Z}^{3}$ with $r>1.5$ then there exists a constant $\mathcal{D}_{4}^{\prime}$, independent of $\mathcal{Z}$, so that

$$
\left|\omega_{k}(t)\right| \leq \frac{\mathcal{D}_{4}^{\prime}}{|k|^{r}}
$$

for all $k \in \mathbb{Z}^{3}$ and $t \geq 0$. 
Theorem 5. Consider the system (20 2 ) with an $\alpha>2.5$ and satisfying assumption S. If the initial data $\left\{\omega_{k}(0)\right\}$ are such that

$$
\left|\omega_{k}(0)\right| \leq \frac{\mathcal{D}_{5}}{|k|^{r}} e^{-\gamma_{5}|k|}
$$

for all $k \in \mathbb{Z}^{3}$ with $r>2$ then there exists constants $\mathcal{D}_{5}^{\prime}<\infty$ and $\gamma_{5}^{\prime}>0$, both independent of $\mathcal{Z}$, so that

$$
\left|\omega_{k}(t)\right| \leq \frac{\mathcal{D}_{5}^{\prime}}{|k|^{r}} e^{-\gamma_{5}^{\prime}|k|}
$$

for all $k \in \mathbb{Z}^{3}$ and $t \geq 0$.

Theorem 6. Consider the system (20 2 ) with an $\alpha>2.5$ and satisfying assumption S. If the initial data $\left\{\omega_{k}(0)\right\}$ are such that

$$
\left|\omega_{k}(0)\right| \leq \frac{\mathcal{D}_{6}}{|k|^{r}}
$$

for all $k \in \mathbb{Z}^{3}$ with $r>2$ then for any $t_{0}>0$ there exists constants $\mathcal{D}_{6}^{\prime}<\infty$ and $\gamma_{6}^{\prime}>0$, both independent of Z, so that

$$
\left|\omega_{k}\left(t_{0}\right)\right| \leq \frac{\mathcal{D}_{6}^{\prime}}{|k|^{r}} e^{-\gamma_{6}^{\prime}|k|}
$$

for all $k \in \mathbb{Z}^{3}$.

Of these three theorems, we will only give the proof of the first. The second two will be the consequence of two more general theorems given below. They apply to all $\alpha>1.5$ but require the additional assumption that the enstrophy of all Galerkin approximations, starting from a given set of initial data, remains uniformly bounded in time. This is not known in general. However, when $\alpha>2.5$, theorem 1 implies this bound. Hence, the two theorems below apply to $(26 \%)$ when $\alpha>2.5$ without any assumption on $\mathcal{E}(t)$. In light of theorem 4 , theorem 7 and 8 respectively yield theorem 5 and 6 when $\alpha>2.5$.

Theorem 7. Let $\left\{u_{k}(t)\right\}$ be a solution to $\left(\mathbb{2}_{z}^{\alpha}\right)$ with $\alpha>1.5$ such that $\sum_{\mathbb{Z}^{3}}\left|\omega_{k}(t)\right|^{2}<\mathcal{E}^{*}<\infty$ for all $t>0$. If $\left|\omega_{k}(0)\right| \leq \frac{\mathcal{D}_{7}}{|k|^{r}}$ for some $\mathcal{D}_{7}<\infty$ and $r>2$ then for any $t_{1}>0$ there exists a $\gamma_{7}>0$ and $\mathcal{D}_{7}^{\prime}<\infty$ such that

$$
\left|\omega_{k}\left(t_{1}\right)\right| \leq \frac{\mathcal{D}_{7}^{\prime}}{|k|^{r}} e^{-\gamma_{7}|k|}
$$

Theorem 8. Let $\left\{u_{k}(t)\right\}$ be a solution to $\left(\mathbb{Q}_{z}^{\alpha}\right)$ with $\alpha>1.5$ such that $\sum_{\mathbb{Z}^{3}}\left|\omega_{k}(t)\right|^{2}<\mathcal{E}^{*}<\infty$ for all $t>0$. If for some $\mathcal{D}_{8}<\infty, \gamma_{8}>0$, and $r>2,\left|\omega_{k}(0)\right| \leq \frac{\mathcal{D}_{8}}{|k|^{r}} e^{-\gamma_{8}|k|}$ then there exists a $\gamma_{8}^{\prime}>0$ and $\mathcal{D}_{8}^{\prime}<\infty$ such that for all $t>0$

$$
\left|\omega_{k}(t)\right| \leq \frac{\mathcal{D}_{8}^{\prime}}{|k|^{r}} e^{-\gamma_{8}^{\prime}|k|}
$$

The above two theorems apply to $(26 \%)$ for $\alpha>1.5$. In particular, this means that they cover the standard Navier-Stokes equation which corresponds to $\alpha=2$. (One can lower the restriction on $\alpha$ to $\alpha>1$ at the cost of raising the restriction on $r$ to $r>3$. Similarly, one lowers the restriction on $r$ to $r>1.5$ at the cost of making $\alpha>2.5$.)

In proving these two theorems, it was necessary to assume that $\sum_{\mathbb{Z}^{3}}\left|\omega_{k}(t)\right|^{2}$ remained uniformly bounded in time. Without such an assumption, we are forced to consider only $\alpha$ which do not correspond to the NavierStokes equation. Notice that theorem $\mathbb{A}$ implies that $\sum_{k \in \mathbb{Z}^{3}}\left|\omega_{k}(t)\right|^{2}<$ const $<\infty$ for all $t>0$ and hence theorems 8 and 8 apply showing that the solution is analytic after $t=0$. 
In proving the above results, it is again convenient to split the system $\left(26 \frac{\alpha}{z}\right)$ into the equations for the real and imaginary parts of $\left\{u_{k}\right\}_{k}$ and $\left\{\omega_{k}\right\}_{k}$. Letting $u_{k}(t)=u_{k}^{(1)}(t)+i u_{k}^{(2)}(t), \omega_{k}(t)=\omega_{k}^{(1)}(t)+i \omega_{k}^{(2)}(t)$, and $g_{k}(t)=g_{k}^{(1)}(t)+i g_{k}^{(2)}(t)$; we obtain

$$
\begin{aligned}
& \frac{d \omega_{k}^{(1)}(t)}{d t}=2 \pi \sum_{\substack{l_{1}+l_{2}=k \\
l_{1}, l_{2} \in \mathcal{Z}}}\left[\left(u_{l_{1}}^{(1)}(t), k\right) \omega_{l_{2}}^{(2)}(t)+\left(u_{l_{1}}^{(2)}(t), k\right) \omega_{l_{2}}^{(1)}(t)-\left(\omega_{l_{1}}^{(2)}(t), k\right) u_{l_{2}}^{(1)}(t)-\left(\omega_{l_{1}}^{(1)}(t), k\right) u_{l_{2}}^{(2)}(t)\right] \\
& \quad-4 \pi^{2} \nu|k|^{\alpha} \omega_{k}^{(1)}(t)+g_{k}^{(1)}(t) \\
& \begin{aligned}
\frac{d \omega_{k}^{(2)}(t)}{d t}=-2 \pi & \sum_{\substack{l_{1}+l_{2}=k \\
l_{1}, l_{2} \in \mathcal{Z}}}\left[\left(u_{l_{1}}^{(1)}(t), k\right) \omega_{l_{2}}^{(1)}(t)-\left(u_{l_{1}}^{(2)}(t), k\right) \omega_{l_{2}}^{(2)}(t)-\left(\omega_{l_{1}}^{(1)}(t), k\right) u_{l_{2}}^{(1)}(t)+\left(\omega_{l_{1}}^{(2)}(t), k\right) u_{l_{2}}^{(2)}(t)\right] \\
& +g_{k}^{(2)}(t)-4 \pi^{2} \nu|k|^{\alpha} \omega_{k}^{(2)}(t)
\end{aligned}
\end{aligned}
$$

Proof of Theorem . By the energy estimate, we know that $\left|u_{k}^{(j)}(t)\right| \leq \sqrt{E^{*}}$ for all $t \geq 0$ and $j=1,2$. Hence, $\left|\omega_{k}^{(j)}(t)\right| \leq|k| \sqrt{E^{*}}$. Fixing a $K_{0}$, set $\mathcal{D}_{4}^{\prime}\left(K_{0}\right)=K_{0} \mathcal{D}_{4}$. With this choice, $\left|\omega_{k}^{(j)}(t)\right| \leq \mathcal{D}_{4}^{\prime}\left(K_{0}\right)$ for all $t \geq 0$, $j=1,2$, and $k \in \mathbb{Z}^{3}$ with $|k| \leq K_{0}$. As before, consider the set

$$
\Omega_{4}\left(K_{0}\right)=\left\{\left(\omega_{k}^{(1)}, \omega_{k}^{(2)}\right)_{k \in \mathbb{Z}^{3}}:\left|\omega_{k}^{(1)}\right| \leq \frac{\mathcal{D}_{4}^{\prime}\left(K_{0}\right)}{|k|^{r}},\left|\omega_{k}^{(2)}\right| \leq \frac{\mathcal{D}_{4}^{\prime}\left(K_{0}\right)}{|k|^{r}} \text { for all } k,|k|>K_{0}\right\} .
$$

We have to show that if $K_{0}$ is taken to be sufficiently large, the vector field points inward along $\partial \Omega_{4}$.

We pick a point on the boundary. For definiteness, we will again consider the case when $\omega_{\bar{k}}^{(1)}=\frac{\mathcal{D}_{4}^{\prime}}{|k|^{r}}$ and $\omega_{\bar{k}}^{(2)} \leq \frac{\mathcal{D}_{4}^{\prime}}{|k|^{r}}$ for some $\bar{k}$ with $|\bar{k}| \geq K_{0}$ and $\omega_{k}^{(j)} \leq \frac{\mathcal{D}_{4}^{\prime}}{|k|^{r}}$ for all other $k$ with $k \neq \bar{k}$. The theorem will be proven if we can show that there exists a $K_{\text {crit }}$, independent of $\mathcal{D}_{4}^{\prime}$, so that if $|\bar{k}| \geq K_{0}>K_{\text {crit }}$ then

$$
\left|2 \pi \sum_{\substack{l_{1}+l_{2}=\bar{k} \\ l_{1}, l_{2} \in \mathcal{Z}}}\left[\left(u_{l_{1}}^{(1)}(t), \bar{k}\right) \omega_{l_{2}}^{(2)}(t)+\left(u_{l_{1}}^{(2)}(t), \bar{k}\right) \omega_{l_{2}}^{(1)}(t)-\left(\omega_{l_{1}}^{(2)}(t), \bar{k}\right) u_{l_{2}}^{(1)}(t)-\left(\omega_{l_{1}}^{(1)}(t), \bar{k}\right) u_{l_{2}}^{(2)}(t)\right]\right|<4 \pi^{2} \nu|\bar{k}|^{\alpha} \omega_{\bar{k}}^{(1)}(t) .
$$

Other boundary points have the same structure so we will only show the details of the calculation for this case.

We need to estimate the summation. The total sum is made of smaller sums which are dominated by sums of the form $\sum_{l_{1}+l_{2}=\bar{k}}\left|u_{l_{1}}^{(a)}\right|\left|\omega_{l_{2}}^{(b)}\right||k|$ with $a, b \in\{1,2\}$. As before, we split this sum into three parts:

$$
\begin{aligned}
& \Sigma_{1}=\sum_{\left|l_{1}\right| \leq\left|\frac{\bar{k}}{2}\right|}\left|u_{l_{1}}^{(a)}\right|\left|\omega_{l_{2}}^{(b)}\right||k| \\
& \Sigma_{2}=\sum_{\left|\frac{\bar{k}}{2}\right|<\left|l_{1}\right| \leq 2|\bar{k}|}\left|u_{l_{1}}^{(a)}\right|\left|\omega_{l_{2}}^{(b)}\right||k| \\
& \Sigma_{3}=\sum_{2|\bar{k}|<\left|l_{1}\right|}\left|u_{l_{1}}^{(a)}\right|\left|\omega_{l_{2}}^{(b)}\right||k|
\end{aligned}
$$

In $\Sigma_{1},\left|l_{2}\right| \geq\left|\frac{\bar{k}}{2}\right|$ and hence

$$
\begin{aligned}
\Sigma_{1} & \leq \frac{\mathcal{D}_{4}^{\prime}}{|\bar{k}|^{2}} 2^{r}|\bar{k}|\left(\sum_{\left|l_{1}\right| \leq\left|\frac{\bar{k}}{2}\right|}\left|u_{l_{1}}^{(a)}\right|^{2}\right)^{\frac{1}{2}}\left(\sum_{\left|l_{1}\right| \leq\left|\frac{\bar{k}}{2}\right|} 1\right)^{\frac{1}{2}} \\
& \leq \frac{\mathcal{D}_{4}^{\prime}}{|\bar{k}|^{r}} 2^{r} \text { (const) } \sqrt{E^{*}}|\bar{k}|^{\frac{5}{2}}
\end{aligned}
$$


The constant is defined by

$$
\left(\sum_{\left|l_{1}\right| \leq\left|\frac{\bar{k}}{2}\right|} 1\right) \leq(\text { const })^{2}|\bar{k}|^{3}
$$

For $\Sigma_{2}$, we know that $\left|l_{2}\right| \leq 3|\bar{k}|$ and $\left|u_{l_{1}}^{(a)}\right| \leq \frac{\mathcal{D}_{4}^{\prime}}{\left|l_{1}\right|^{r+1}}$ which gives

$$
\begin{aligned}
\Sigma_{2} & \leq \frac{\mathcal{D}_{4}^{\prime}}{|\bar{k}|^{r}} 2^{r} \frac{2}{|k|}|k| \sum_{\left|l_{2}\right| \leq 3|\bar{k}|}\left|\omega_{l_{2}}^{(b)}\right| \leq \frac{\mathcal{D}_{4}^{\prime}}{|\bar{k}|^{r}} 2^{r+1} \sum_{\left|l_{2}\right| \leq 3|\bar{k}|}\left|l_{2}\right|\left|u_{l_{2}}^{(a)}\right| \\
& \leq \frac{\mathcal{D}_{4}^{\prime}}{|\bar{k}|^{r}} 2^{r+1} 3|\bar{k}|\left(\sum_{\left|l_{2}\right| \leq 3|\bar{k}|}\left|u_{l_{1}}^{(a)}\right|^{2}\right)^{\frac{1}{2}}\left(\sum_{\left|l_{2}\right| \leq 3|\bar{k}|} 1\right)^{\frac{1}{2}} \\
& \leq \frac{\mathcal{D}_{4}^{\prime}}{|\bar{k}|^{r}} 2^{r+1} 3 \text { (const) } \sqrt{E^{*}|\bar{k}|^{\frac{5}{2}}} .
\end{aligned}
$$

Here the constant is the analogue of the constant in the estimation of $\Sigma_{1}$. For $\Sigma_{3}$, we know that $\left|l_{2}\right| \geq|\bar{k}|$ and thus

$$
\begin{aligned}
\Sigma_{3} & \leq|\bar{k}|\left(\sum_{\left|l_{1}\right| \geq 2|\bar{k}|}\left|u_{l_{1}}\right|^{2}\right)^{\frac{1}{2}}\left(\sum_{\left|l_{2}\right| \geq|\bar{k}|}\left|\omega_{l_{2}}\right|^{2}\right)^{\frac{1}{2}} \leq|\bar{k}| \mathcal{D}_{4}^{\prime} \sqrt{E^{*}}\left(\sum_{\left|l_{2}\right| \geq|\bar{k}|} \frac{1}{\left|l_{2}\right|^{2 r}}\right)^{\frac{1}{2}} \\
& \left.\leq|\bar{k}| \mathcal{D}_{4}^{\prime} \sqrt{E^{*}} \frac{(\text { const })}{|\bar{k}|^{r-\frac{3}{2}}} \leq \frac{\mathcal{D}_{4}^{\prime}}{|\bar{k}|^{r}} \text { const }\right) \sqrt{E^{*}}|\bar{k}|^{\frac{5}{2}} .
\end{aligned}
$$

Collecting the three estimates together we see that there is a constant, depending only on $r$, so that

$$
\sum_{l_{1}+l_{2}=\bar{k}}\left|u_{l_{1}}^{(a)}\right|\left|\omega_{l_{2}}^{(b)}\right||k| \leq \text { (const) } \frac{\mathcal{D}_{4}^{\prime}}{|\bar{k}|^{r}} \sqrt{E^{*}|\bar{k}|^{\frac{5}{2}}}
$$

Using this estimate, we see that the condition in (伍) will hold if

$$
8 \pi \text { (const) } \sqrt{E^{*}}|\bar{k}|^{\frac{5}{2}} \frac{\mathcal{D}_{4}^{\prime}}{|\bar{k}|^{r}}<4 \pi^{2} \nu|\bar{k}|^{\alpha} \frac{\mathcal{D}_{8}^{\prime}}{|\bar{k}|^{r}} .
$$

Since $\alpha>\frac{5}{2}$, this will hold for all $\bar{k}$ sufficiently large; this sets the level of $K_{\text {crit }}$. Notice that it does not depend on $\mathcal{D}_{8}^{\prime}$ as was required.

Proof of Theorem 1 . The proof of this theorem is similar to the proof of theorem 3 . From the assumptions, we know that $\left|\omega_{k}(t)\right| \leq \sqrt{\sum_{\mathbb{Z}^{3}}\left|\omega_{l}(t)\right|^{2}}<\sqrt{\mathcal{E}^{*}}$ for all $t>0$. We set $a_{k}^{(j)}(t)=u_{k}^{(j)} e^{\gamma_{7} t|k|}$ and $b_{k}^{(j)}(t)=\omega_{k}^{(j)} e^{\gamma_{7} t|k|}$ for $j=1,2$, where $\gamma_{7}$ is a constant we will set later.

Set $\mathcal{D}_{7}^{\prime}=2 \max \left(\sqrt{\mathcal{E}^{*}}, \mathcal{D}_{7}\right)$. Fixing a $K_{0}$, choose $\gamma_{7}\left(K_{0}\right)$ so that for all $t \in\left[0, t_{1}\right], j \in\{1,2\}$, and $k$ with $|k| \leq K_{0}$, one has $\left|b_{k}^{(j)}(t)\right| \leq \frac{\mathcal{D}_{7}^{\prime}}{|k|^{r}}$. Notice that by the assumption on the initial conditions, we have $\left|b_{k}^{(j)}(0)\right| \leq \frac{\mathcal{D}_{7}^{\prime}}{|k|^{r}}$ for all $k$. Consider the set,

$$
\Omega_{7}\left(K_{0}\right)=\left\{\left(b_{k}^{(1)}, b_{k}^{(2)}\right)_{k \in \mathbb{Z}^{2} \backslash 0} \text { with }\left|b_{k}^{(j)}\right| \leq \frac{\mathcal{D}_{7}^{\prime}}{|k|^{r}} \text { for } j=1,2 \text { and }|k|>K_{0}\right\} .
$$

As before we will show that, if $K_{0}$ is chosen large enough, a point starting in $\Omega_{7}$ cannot leave $\Omega_{7}$ because the vector field along $\partial \Omega_{7}$ is pointing inward.

We pick a boundary point. For simplicity, we pick the point where $b_{\bar{k}}^{(1)}=\frac{\mathcal{D}_{7}^{\prime}}{|k|^{r}}$ and all other variables satisfy the inequalities defining $\Omega_{7}$. In terms of the new variables, the relevant equation of motion reads

$$
\begin{aligned}
\frac{d b_{k}^{(1)}(t)}{d t} & =\left(\gamma_{7}|k|-4 \pi^{2} \nu|k|^{\alpha}\right) b_{k}^{(1)}(t)-2 \pi \sum_{\substack{l_{1}+l_{2}=k \\
l_{1}, l_{2} \in \mathcal{Z}}}\left[\left(a_{l_{1}}^{(1)}(t), k\right) b_{l_{2}}^{(2)}(t)\right. \\
& \left.+\left(a_{l_{1}}^{(2)}(t), k\right) b_{l_{2}}^{(1)}(t)-\left(b_{l_{1}}^{(2)}(t), k\right) a_{l_{2}}^{(1)}(t)-\left(b_{l_{1}}^{(1)}(t), k\right) a_{l_{2}}^{(2)}(t)\right] \frac{e^{-\gamma_{7} t\left|l_{1}\right|} e^{-\gamma_{7} t\left|l_{2}\right|}}{e^{-\gamma_{7} t|k|}} .
\end{aligned}
$$


Since $\left|a_{k}^{(j)}(t)\right|=\frac{\left|b_{k}^{(j)}(t)\right|}{|k|}$, to insure that the vector field points inward it is sufficient to show that

$$
\begin{aligned}
2 \pi \sum\left|b_{l_{1}}^{(1)}\right|\left|b_{l_{2}}^{(2)}\right| \frac{|\bar{k}|}{\left|l_{1}\right|}+\left|b_{l_{1}}^{(2)}\right|\left|b_{l_{2}}^{(1)}\right| \frac{|\bar{k}|}{\left|l_{1}\right|}+\left|b_{l_{1}}^{(2)} \|\right| b_{l_{2}}^{(1)} \mid \frac{|\bar{k}|}{\left|l_{2}\right|} & +\left|b_{l_{1}}^{(1)}\right|\left|b_{l_{2}}^{(2)}\right| \frac{|\bar{k}|}{\left|l_{2}\right|} \\
& <\left(4 \pi^{2} \nu|\bar{k}|^{\alpha}-\gamma_{7}|\bar{k}|\right) \frac{\mathcal{D}_{7}^{\prime}}{|\bar{k}|^{r}} .
\end{aligned}
$$

Each of the terms in the above sum can be estimated with the aid of lemma 11. This transforms the previous condition into

$$
8 \pi \text { (const) }\left(2^{r}|\bar{k}|+2^{r+1}(6|\bar{k}|+1)^{\frac{3}{2}}+\frac{1}{2}|\bar{k}|^{2-r}\right) \frac{\left(\mathcal{D}_{7}^{\prime}\right)^{2}}{|\bar{k}|^{r}}<\left(4 \pi^{2} \nu|\bar{k}|^{\alpha}-\gamma_{7}|\bar{k}|\right) \frac{\mathcal{D}_{7}^{\prime}}{|\bar{k}|^{r}} .
$$

By picking $K_{0}$ large enough, we can force this condition to hold. The fact that $\gamma_{7}$ depends on $K_{0}$ is not a problem since it decreases as $K_{0}$ increases.

This establishes that the vector field points inward along the boundary of $\Omega_{7}$ for all times in the interval $\left[0, t_{1}\right]$. Thus at time $t_{1}$, the trajectory is still in $\Omega_{7}$. By returning to the original variables, we have the desired estimate at time $t_{1}$.

Proof of Theorem 8 . The proof of this theorem begins as the above theorem and then proceeds as the proof of theorem 2. We change variables to $a_{k}^{(j)}(t)=u_{k}^{(j)} e^{\gamma_{8}|k|}$ and $b_{k}^{(j)}(t)=\omega_{k}^{(j)} e^{\gamma_{8}|k|}$. We use the assumption on $\sqrt{\sum_{\mathbb{Z}^{3}}\left|\omega_{l}(t)\right|^{2}}$ to control the lower modes. Then we use the estimates from lemma 1 to control the nonlinearity. We omit the details.

\section{Acknowledgements}

The authors thank W.E., C. Fefferman, U. Frisch, G. Gallavotti, J. Mather, V. I. Judovich, F. Planchon, P. Sarnak, T. Spencer, T. Suidan, J. Vinson, and V. Yakhot for useful discussions. The second author thanks NSF (grant DMS-97067994) for financial support.

\section{References}

[CF88] Peter Constantin and Ciprian Foiaş. Navier-Stokes Equations. University of Chicago Press, Chicago, 1988.

[CM93] Alexandre J. Chorin and Jerrold E. Marsden. A mathematical introduction to fluid mechanics, volume 4 of Texts in Applied Mathematics. Springer-Verlag, New York, third edition, 1993.

[DG95] Charles R. Doering and J. D. Gibbon. Applied analysis of the Navier-Stokes equations. Cambridge Texts in Applied Mathematics. Cambridge University Press, Cambridge, 1995.

[DT95] Charles R. Doering and Edriss S. Titi. Exponential decay rate of the power spectrum for solutions of the Navier-Stokes equations. Phys. Fluids, 7(6):1384-1390, 1995.

[FT89] C. Foias and R. Temam. Gevrey class regularity for the solutions of the Navier-Stokes equations. J. Funct. Anal., 87(2):359-369, 1989.

[Gal96] G.Gallavotti. Ipotesi per una introduzione alla Meccanica Dei Fluidi Gruppo Nazionale Di Fisica Matematica, 1996.

[Hop51] Eberhard Hopf. Über die Anfangswertaufgabe für die hydrodynamischen Grundgleichungen. Math. Nachr., 4:213-231, 1951.

[Kre88] Heinz-Otto Kreiss. Fourier expansions of the solutions of the Navier-Stokes equations and their exponential decay rate. In Analyse mathématique et applications, pages 245-262. Gauthier-Villars, Paris, 1988. 
[Lad69] O. A. Ladyzhenskaya. The Mathematical Theory of Viscous Incompressible Flow. Gordon and Breach, New York, 1969.

[Ler34] J. Leray. Essai sur le mouvement d'un liquide visqueux emplissant l'espace. Acta Math., 63:193-248, 1934.

[Tem79] Roger Temam. Navier-Stokes equations:Theory and numerical analysis, volume 2 of Studies in Mathematics and its Applications. North-Holland Publishing Co., Amsterdam-New York, revised edition, 1979.

[Tem95] Roger Temam. Navier-Stokes equations and nonlinear functional analysis, volume 66 of CBMS-NSF Regional Conference Series in Applied Mathematics. Society for Industrial and Applied Mathematics (SIAM), Philadelphia, PA, second edition, 1995.

[Yud89] V. I. Yudovich. The linearization method in hydrodynamical stability theory. American Mathematical Society, Providence, RI, 1989. Translated from the Russian by J. R. Schulenberger. 